Case Report

\title{
Acute Spontaneous Coronary Artery Thrombosis as Initial Presentation of HIV Infection in a Young Man
}

\author{
James Kayima, ${ }^{1,2}$ Wilson Nyakoojo, ${ }^{2}$ Damalie Nakanjako, ${ }^{1,3}$ Marco A. Costa, ${ }^{4}$ \\ Christopher T. Longenecker, ${ }^{4}$ and Daniel I. Simon ${ }^{4}$ \\ ${ }^{1}$ Department of Medicine, School of Medicine, College of Health Sciences, Makerere University, P.O. Box 7072, Kampala, Uganda \\ ${ }^{2}$ Uganda Heart Institute, Ward 1C, Mulago Hospital Complex, P.O. Box 7051, Kampala, Uganda \\ ${ }^{3}$ Infectious Disease Institute, Makerere University, P.O. Box 22418, Kampala, Uganda \\ ${ }^{4}$ Harrington Heart and Vascular Institute, University Hospitals Case Medical Center, Case Western Reserve University, \\ School of Medicine, 11100 Euclid Avenue, Cleveland, OH 44106, USA
}

Correspondence should be addressed to James Kayima; jkkayima@gmail.com

Received 4 December 2014; Accepted 11 February 2015

Academic Editor: Expedito E. Ribeiro

Copyright (C) 2015 James Kayima et al. This is an open access article distributed under the Creative Commons Attribution License, which permits unrestricted use, distribution, and reproduction in any medium, provided the original work is properly cited.

Introduction. The presentation of acute coronary syndrome (ACS) in young HIV patients may be atypical with different pathophysiological and clinical features. Acute coronary thrombosis, as a presentation of acute coronary syndrome in young patients with HIV, raises diagnostic and treatment challenges. Case Presentation. We describe a case of a 33-year-old African man, without traditional atherosclerotic risk factors, who presented with chest pain of acute onset. Emergent coronary angiography revealed extensive thrombus in the left anterior descending coronary artery with no evidence of atherosclerosis in this or other coronary vessels. Plaque and/or thrombus prolapse through the stent was noted following percutaneous coronary intervention (PCI). Resolution of chest pain and improvement in ST-segment elevation was noted after the procedure. A diagnosis of HIV infection was made during the workup for HIV infection. Conclusion. In young patients without traditional risk factors, HIV infection is a possible etiological factor for spontaneous coronary artery thrombosis. Percutaneous coronary intervention in patients with this presentation may be compounded with atherothrombotic complications. The likely pathophysiological pathway is superficial endothelial cell denudation as a result of chronic inflammation and immune activation.

\section{Introduction}

An increased risk of myocardial infarction has been documented in HIV-infected patients since the advent of efficacious antiretroviral therapy (ART) that prolongs life. An increased prevalence of traditional risk factors (e.g., smoking), uncontrolled viral replication, and metabolic toxicities of ART have been proposed as underlying drivers of atherosclerosis and risk for ACS [1], yet few studies have been conducted in sub-Saharan Africa [2]. Spontaneous coronary thrombosis in young HIV individuals has been described in patients on antiretroviral therapy [3,4]. We report a somewhat rare case of a 33-year-old man with previously undiagnosed HIV-infection who emergently presented with an anteroseptal ST-segment-elevation myocardial infarction (STEMI). Coronary angiograms revealed extensive thrombus in the left anterior descending artery with minimal angiographic coronary artery disease and no spontaneous coronary artery dissection. This presentation may pose challenges in management.

\section{Case Presentation}

A 33-year-old man was referred urgently to our medical facility. He reported a history of intermittent chest pain over the preceding two weeks. He attributed the chest pain to musculoskeletal issues due to lack of physical activity, and he had resorted to aerobic exercise sessions for relief. Two hours before presentation to the referring medical unit, he developed severe retrosternal chest pressure accompanied by dyspnea. An ECG performed at the referring hospital 


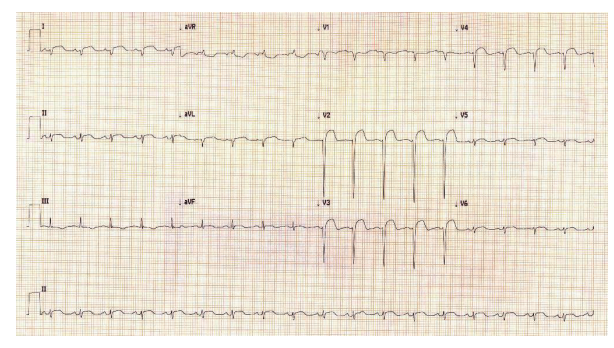

FIGURE 1: ECG on admission showing anterolateral ST elevation.

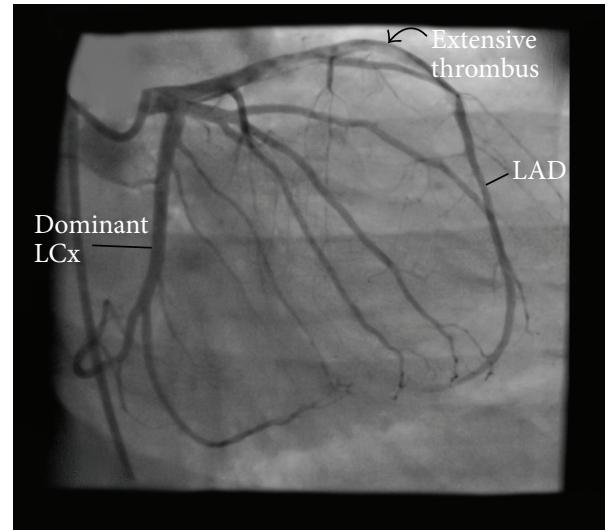

FIGURE 2: Preintervention angiographic image showing extensive LAD thrombosis. LAD: left anterior descending coronary artery; LCx: left circumflex coronary artery.

revealed ST-segment elevation V1-V6 consistent with an anterolateral STEMI and he was promptly transferred to our cardiac care unit (Figure 1).

His past medical history was negative for hypertension, diabetes, dyslipidemia, or smoking. He had no family history of cardiac problems or sudden death. Social history was negative for recreational drug use.

Physical examination revealed an overweight young man $(\mathrm{BMI}=27)$ who was uncomfortable secondary to chest pain. He was afebrile. Blood pressure was $141 / 81 \mathrm{mmHg}$ and equal in arms, heart rate 62 beats/min, and respiratory rate 16 breaths/min. The chest was clear to auscultation. He had no jugular venous distention and cardiac auscultation revealed normal S1 and S2 with no S3 or murmurs.

Because the patient had persistent chest pain and STsegment-elevation, a decision was made to take him for urgent left heart catheterization, coronary angiography, and primary PCI. The patient was pretreated with aspirin $325 \mathrm{mg}$, prasugrel $60 \mathrm{mg}$ oral load, and unfractionated heparin 4,000 units IV bolus. During preparation for the procedure, the initial laboratory data returned. These revealed creatinine of $74 \mu \mathrm{g} / \mathrm{mL}$ (unknown baseline), a normal complete blood count (CBC), and coagulation study. Liver function tests were abnormal for alkaline phosphatase of 154 (35-105), alanine aminotransferase (ALT) 39 (7-35), and aspartate aminotransferase (AST) 72 (13-35). An initial set of cardiac enzymes were mildly elevated with a creatine kinase myocardial band $(\mathrm{CKMB})$ mass of $13.00 \mathrm{ng} / \mathrm{mL}$ (normal <4) and troponin of

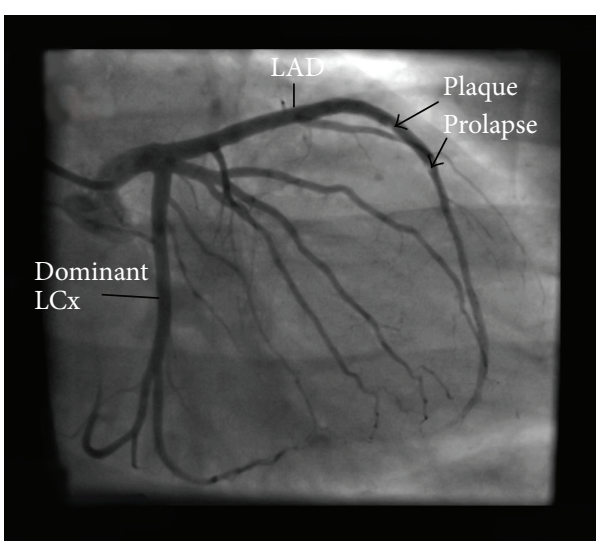

FIgURE 3: Postintervention angiographic image showing plaque prolapse. LAD: left anterior descending coronary artery; LCx: left circumflex coronary artery.

$0.1 \mathrm{ng} / \mathrm{mL}$ (normal 0.0 to 0.04 ). His lipid panel was within normal limits, and a urinalysis showed proteinuria. Screening for infection, which is routine at our facility, revealed normal hepatitis B and C serology. The HIV antibody test was positive. His CD4+ count was 643 cells/ $\mu \mathrm{L}$.

Coronary angiography revealed extensive, nonocclusive thrombus extending from proximal to mid left anterior descending (LAD) coronary artery with no significant atherosclerosis in this or other vessels (Figure 2). A decision was made to intervene on the LAD lesion. The left coronary artery was engaged with 6F EBU 3.5 guide catheter. A 0.014 Asahi Prowater guide wire was used to cross the thrombotic lesion and advanced into the distal LAD. Three thrombectomy passes were made with an Export catheter (Medtronic, Minneapolis, Minnesota, USA) yielding moderate amount of red (i.e., red blood cell-rich) and white (i.e., plateletrich) thrombi. Follow-up angiography revealed large residual thrombus burden. Two Resolute Integrity (Medtronic) drug eluting stents $(3.5 \times 26$ and $3.0 \times 30)$ were deployed in an overlapping fashion to cover the thrombotic lesion. The stents were postdilated with a $3.5 \times 20$ noncompliant balloon to 18-22 atmosphere of pressure. Follow-up angiography after intracoronary nitroglycerin revealed TIMI II flow and mild plaque and/or thrombus prolapse through the distal stent (Figure 3). At the conclusion of the case, the patient was pain free with near ST-segment resolution.

The patient was then admitted to the coronary care unit for monitoring and post-PCI care. Echocardiography revealed overall preserved left ventricular function with anterior hypokinetic and left ventricular ejection fraction of $55 \%$. Treatment with dual antiplatelet therapy (aspirin $81 \mathrm{mg}$ and prasugrel $10 \mathrm{mg}$ ), enalapril, and rosuvastatin was instituted. He was counselled regarding his HIV status and the infectious disease team was engaged to make arrangements for outpatient HIV care.

\section{Discussion}

We report an uncommon case of a patient previously unaware of his HIV status, who presented with STEMI secondary to 
large thrombus burden in the LAD. HIV infection is thought to be a risk factor for MI with an adjusted hazard ratio of 1.5-2 [5]. A higher prevalence of traditional coronary artery disease risk factors in HIV, viral specific factors, and the toxicity of ART have been proposed as pathophysiologic factors promoting atherosclerosis in HIV populations [6]. As a result, MI may occur at younger ages among HIV-infected compared to uninfected populations.

While premature atherosclerosis has been reported in most HIV cases with ACS, there have been very few reported cases of young HIV patients with coronary artery thrombosis with no significant angiographic coronary artery disease. Previously documented cases are notable for known HIV infection with concomitant antiretroviral treatment $[3,4]$. In our patient, whose diagnosis of HIV infection was made in the STEMI setting, the duration of HIV infection could not be ascertained.

The differential diagnosis for $\mathrm{MI}$ in the absence of atherosclerosis includes, among others, coronary artery dissection, superficial endothelial cell erosion (typically associated with younger age, female gender, and diabetes), and coronary artery embolism from left atrial appendage or mural thrombus, endocarditis, and paradoxical fat emboli [7]. In this case, there was no evidence for coronary artery dissection or embolism from subclinical endocarditis or previously unrecognized intracardiac thrombus. To the best of our knowledge, there is no clear association between HIV infection and superficial endothelial cell erosion, which is an angioscopic or pathologic diagnosis.

HIV-associated chronic inflammation and immune activation have been shown to be potential risk factors for cardiovascular disease [8]. The resultant endothelial dysfunction, typically assessed as defective endothelial-dependent vasodilatation of the brachial artery, may be the mechanistic link between HIV infection and acute atherothrombotic events. In this setting, apoptosis of endothelial cells may contribute to desquamation of endothelial cells in areas of superficial erosion [9]. Recent data support the notion that HIV infection itself rather than antiretroviral therapy induces endothelial dysfunction with both micro- and macrovascular abnormalities correlating with CD4 counts in various studies [10]. However, data associating low CD4+ T-cell counts with major adverse cardiovascular events is mixed [11]. In the present case, the patient was antiretroviral therapy naïve, albeit with a relatively spared immune system as evidenced by a high CD 4 count of 643 cells $/ \mu \mathrm{L}$.

ACS due to extensive coronary artery thrombus confers significant treatment challenges. Slow flow and no-reflow secondary to distal embolization are associated with periprocedural infarction and increased mortality. We employed aspiration thrombectomy and direct deployment of a stent to minimize distal embolization in our patient, but it is noteworthy that final angiographic flow was not normal (i.e., TIMI 2 grade flow). The direct stenting technique has been shown to reduce distal embolization by avoiding thrombus disintegration during predilation and using the stent as a scaffolding device to entrap thrombus [12]. When plaque and/or thrombus prolapse occurs, it is controversial whether additional balloon inflations and stent deployment are required. Intravascular imaging-guided studies suggest that endogenous thrombus dissolution typically occurs with no increase in stent thrombosis rates or neointimal formation compared to those without thrombus or plaque prolapse [13].

\section{Conclusion}

This case illustrates an ACS caused by spontaneous LAD thrombosis in a patient with HIV infection. It is likely that the pathogenesis for this event was related to increased risk of thrombosis associated with inflammation, immune activation, and thrombophilia as a result of uncontrolled HIV infection. It is also intriguing to speculate whether this patient experienced superficial endothelial cell erosion as a consequence of HIV infection. Management of the patient was challenging due to high thrombus burden. As primary PCI becomes a viable reperfusion option for STEMI in subSaharan Africa, there is a unique opportunity to further investigate the mechanisms of ACS in the HIV-infected population.

\section{Consent}

Written informed consent was obtained from the patient for publication of this case report and accompanying images.

\section{Disclaimer}

The contents of this paper are solely the responsibility of the authors and do not necessarily represent the official views of the supporting offices.

\section{Conflict of Interests}

The authors declare that they have no competing interests.

\section{Authors' Contribution}

James Kayima and Wilson Nyakoojo played a central role in management of the patient and performed percutaneous coronary intervention. James Kayima, Damalie Nakanjako, Christopher T. Longenecker, Marco A. Costa, and Daniel I. Simon were major contributors in the writing and review of the paper. James Kayima, Damalie Nakanjako, and Christopher T. Longenecker conceived the study and participated in its design. All the authors read and approved the final paper.

\section{Acknowledgments}

The authors thank the catheterization laboratory and intensive care nursing staff of the Uganda Heart Institute for their role in the management of the patient. This work was supported by Grant R24TW008861 (MEPI-Linked: Building Capacity for Cardiovascular Research and Training in Uganda) funded by Office of the United States Global Aids Coordinator, National Institutes of Health, and Health Resources and Services Administration. 


\section{References}

[1] E. A. Eugenin, S. Morgello, M. E. Klotman et al., "Human immunodeficiency virus (HIV) infects human arterial smooth muscle cells in vivo and in vitro: implications for the pathogenesis of HIV-mediated vascular disease," The American Journal of Pathology, vol. 172, no. 4, pp. 1100-1111, 2008.

[2] I. Ssinabulya, J. Kayima, C. Longenecker et al., "Subclinical atherosclerosis among HIV-infected adults attending HIV/AIDS care at two large ambulatory HIV clinics in Uganda," PLoS ONE, vol. 9, no. 2, Article ID e89537, 2014.

[3] M. Çilingiroğlu, N. Lather, A. Youseff, and T. Helmy, "STelevation myocardial infarction due to a spontaneous thrombus in the left anterior descending artery in a young HIV-infected patient," Archives of the Turkish Society of Cardiology, vol. 39, no. 4, pp. 308-311, 2011.

[4] J. Ayers, R. Mandell, K. Sanghvi, R. Aboujaoude, and D. H. Hsi, "Acute coronary thrombosis and multiple coronary aneurysms in a 22-year-old man with the human immunodeficiency virus," Texas Heart Institute Journal, vol. 41, no. 2, pp. 208-211, 2014.

[5] M. S. Freiberg, C.-C. H. Chang, L. H. Kuller et al., "HIV infection and the risk of acute myocardial infarction," JAMA Internal Medicine, vol. 173, no. 8, pp. 614-622, 2013.

[6] N. Friis-Møller, R. Weber, P. Reiss et al., "Cardiovascular disease risk factors in HIV patients-association with antiretroviral therapy. Results from the DAD study," AIDS, vol. 17, no. 8, pp. 1179-1193, 2003.

[7] S. Agewall, L. Eurenius, C. Hofman-Bang et al., "Myocardial infarction with angiographically normal coronary arteries," Atherosclerosis, vol. 219, no. 1, pp. 10-14, 2011.

[8] D. Nakanjako, J. Otiti-Sengeri, I. Ssewanyana et al., "CD4 Tcell activation and reduced regulatory $\mathrm{T}$-cell populations are associated with early development of cataracts among HIVinfected adults in Uganda," Immunology Letters, vol. 161, no. 1, pp. 44-49, 2014.

[9] N. T. Funderburg, D. A. Zidar, C. Shive et al., "Shared monocyte subset phenotypes in HIV-1 infection and in uninfected subjects with acute coronary syndrome," Blood, vol. 120, no. 23, pp. 4599-4608, 2012.

[10] D. Francisci, S. Giannini, F. Baldelli et al., "HIV type 1 infection, and not short-term HAART, induces endothelial dysfunction," AIDS, vol. 23, no. 5, pp. 589-596, 2009.

[11] C. T. Longenecker and V. A. Triant, "Initiation of antiretroviral therapy at high CD4 cell counts: does it reduce the risk of cardiovascular disease?" Current Opinion in HIV \& AIDS, vol. 9, no. 1, pp. 54-62, 2014.

[12] C. Loubeyre, M.-C. Morice, T. Lefèvre, J.-F. Piéchaud, Y. Louvard, and P. Dumas, "A randomized comparison of direct stenting with conventional stent implantation in selected patients with acute myocardial infarction," Journal of the American College of Cardiology, vol. 39, no. 1, pp. 15-21, 2002.

[13] H. Futamatsu, M. Sabaté, D. J. Angiolillo et al., "Characterization of plaque prolapse after drug-eluting stent implantation in diabetic patients: a three-dimensional volumetric intravascular ultrasound outcome study," Journal of the American College of Cardiology, vol. 48, no. 6, pp. 1139-1145, 2006. 


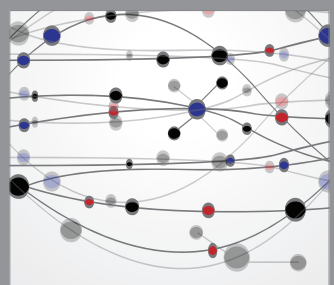

The Scientific World Journal
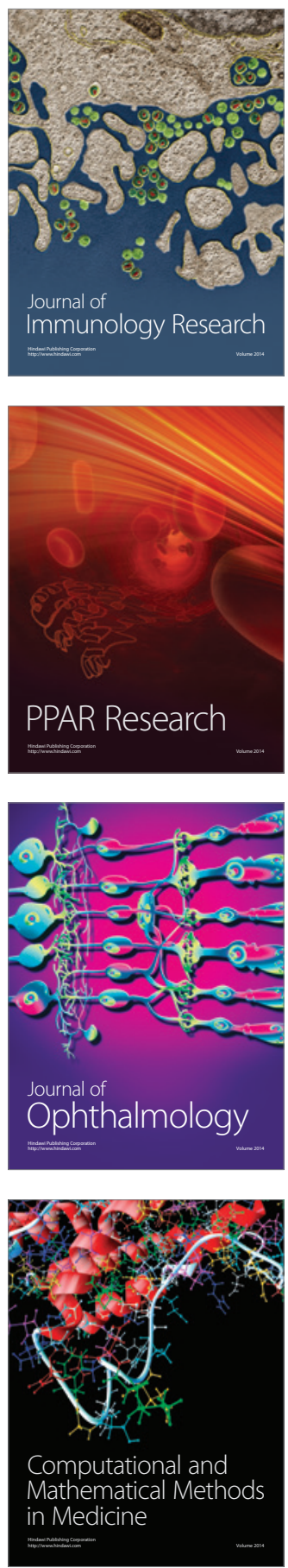

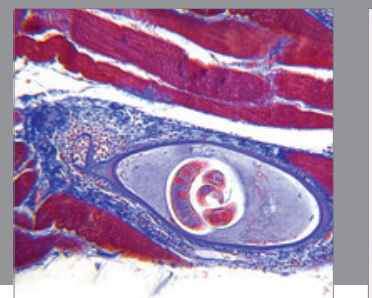

Gastroenterology

Research and Practice
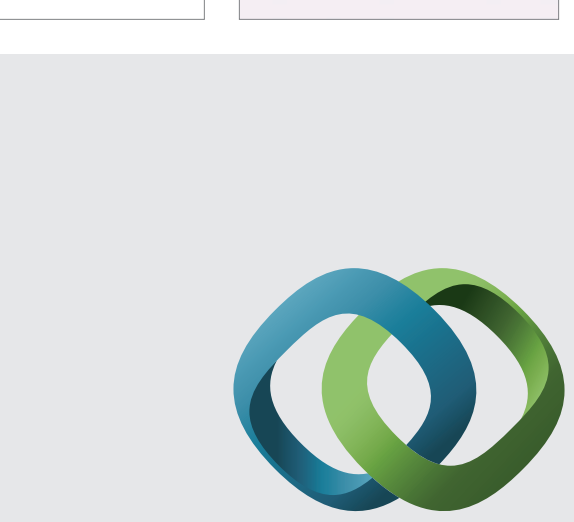

\section{Hindawi}

Submit your manuscripts at

http://www.hindawi.com
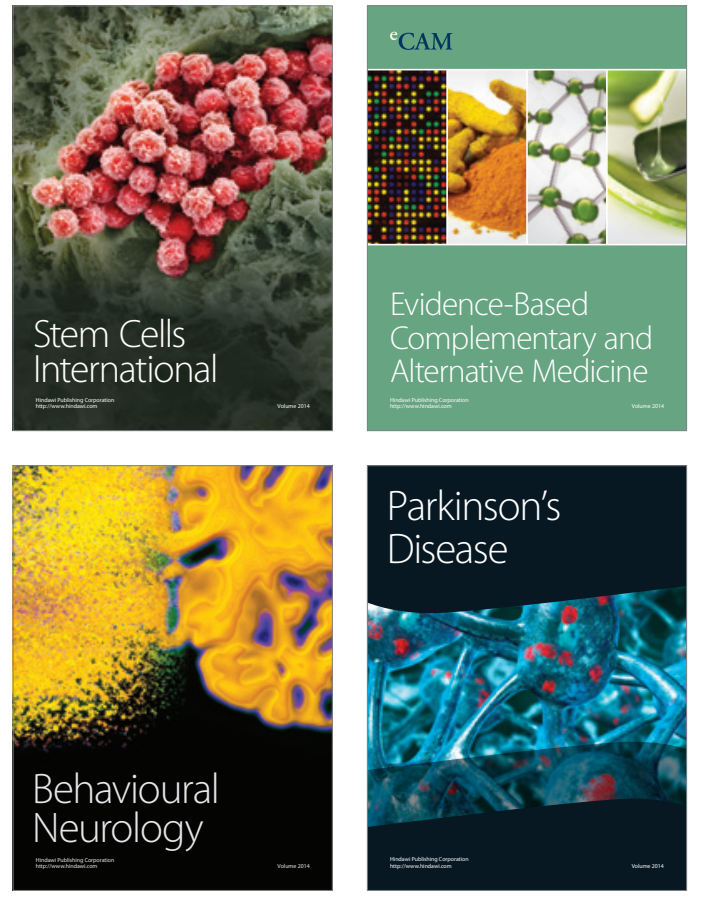
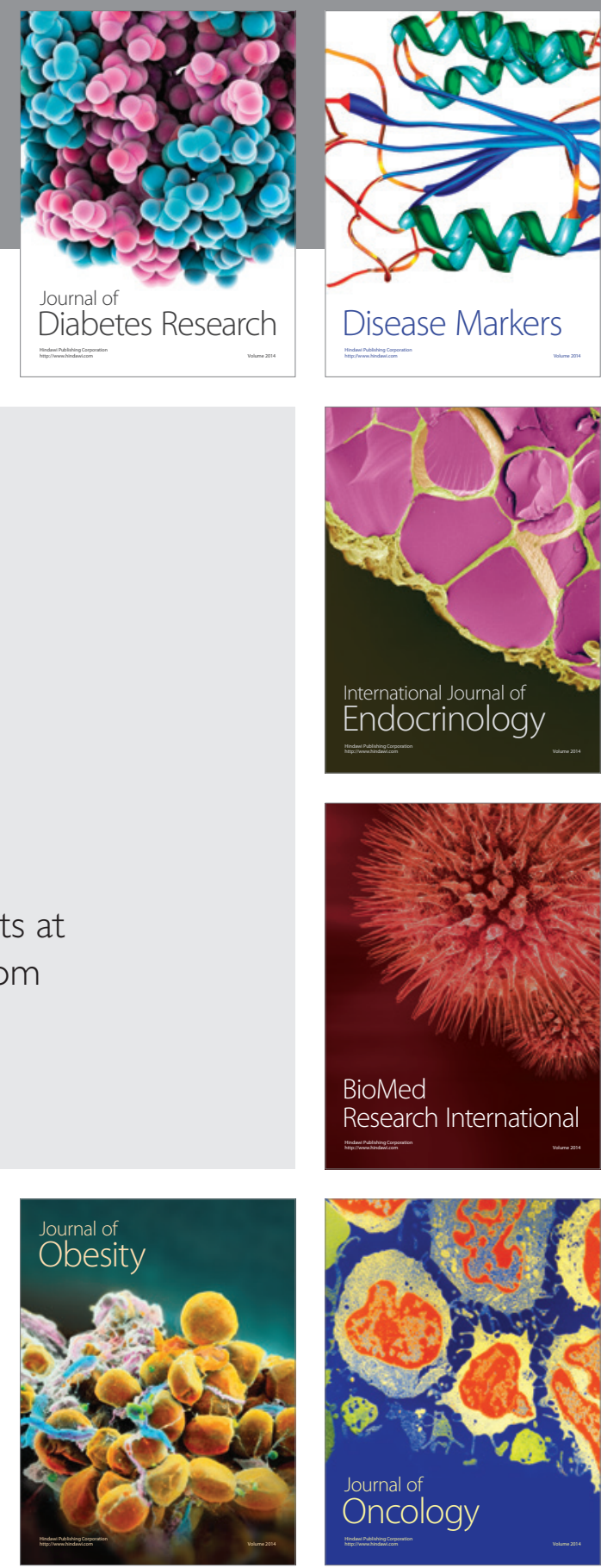

Disease Markers
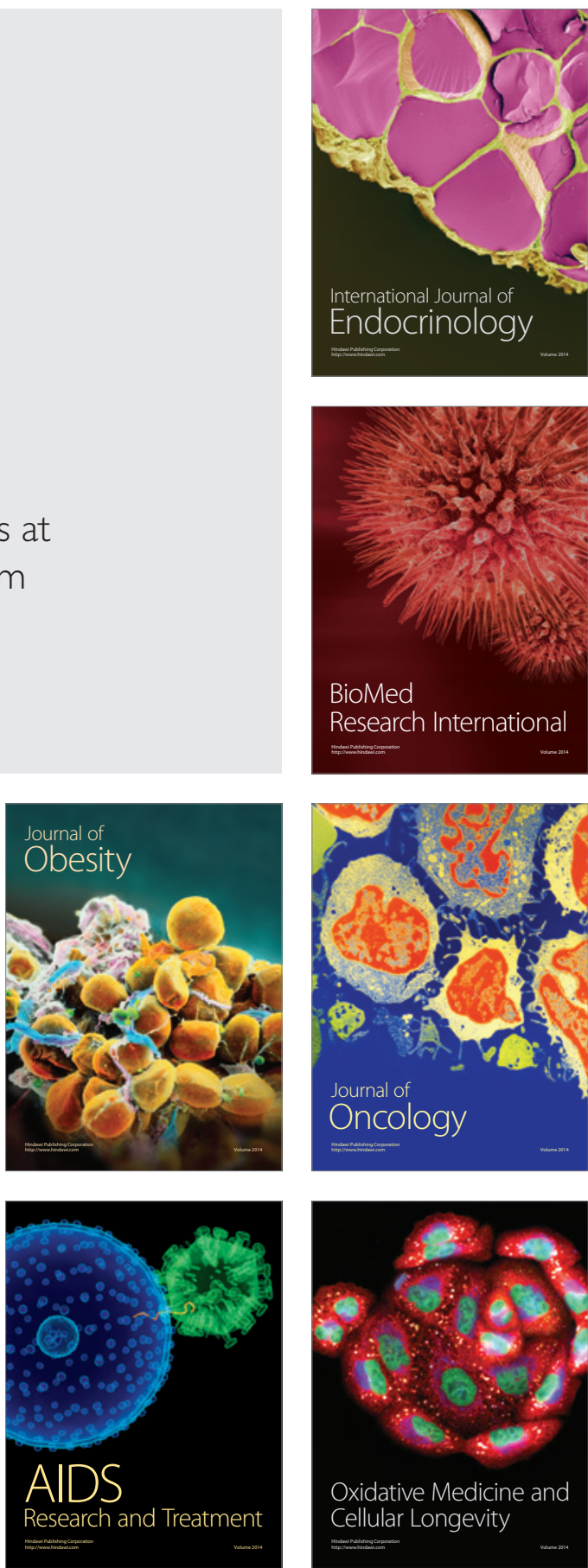